\title{
Joint measure matrix and channel estimation for millimeter-wave massive MIMO with hybrid precoding
}

\author{
Shufeng $\mathrm{Li}^{i^{*}} \mathbb{D}$, Baoxin Su${ }^{1}$, Libiao Jin ${ }^{1^{*}}$, Mingyu Cai ${ }^{1}$ and Hongda $\mathrm{Wu}^{2}$
}

\begin{abstract}
Millimeter-wave (mmWave) massive multiple-input multiple-output (MIMO) with hybrid precoding is a promising technology for future $5 \mathrm{G}$ wireless communications. Channel estimation for the millimeter-wave (mmWave) MIMO systems with hybrid precoding can be performed by estimating the path directions of the channel and corresponding path gains. This paper considers joint measure matrix and channel estimation for a massive MIMO system. By exploiting the sparsity of a massive MIMO system, a channel estimation scheme based on a Toeplitz-structured measure matrix and complete complementary sequence (CC-S) is proposed. Moreover, analytic studies show that the measurement matrix based on CC-S yields either optimal performance or feasibility in practice than an independent identically distributed Gaussian matrix. The performance of the scheme is shown with numerical examples.
\end{abstract}

Keywords: Measurement matrix, Channel estimation, Hybrid precoding millimeter-wave, Massive MIMO

\section{Introduction}

Millimeter-wave (mmWave) communication has been recognized as a promising technology for future 5G wireless communications due to the abundant frequency spectrum resource in a mmWave band [1]. However, the severe signal propagation loss compared with conventional microwave frequencies makes this band just useful for indoor scenarios. Fortunately, the combination of mmWave with short wavelength and massive multipleinput multiple-output (MIMO) array compensate path loss and provide a high degree of spatial freedom to substantially enhance the system's throughput, spectral efficiency, which makes cellular communication on mmWave band practical $[2,3]$. Unfortunately, the mismatch between the number of expensive radio frequency (RF) chain and the large number of antenna makes the conventional full digital precoding impractical. To reduce the overall hardware cost and power consumption, a hybrid analogdigital precoding scheme is proposed, which means that analog precoding is realized by using phase shifters or

\footnotetext{
* Correspondence: shufeng_2004@163.com; libiao@cuc.edu.cn 'school of Information and Communication Engineering, Communication University of China, Beijing 100024, People's Republic of China Full list of author information is available at the end of the article
}

switches in the RF domain and the digital precoding is implemented in the baseband domain as in conventional MIMO [4-6].

For mmWave massive MIMO system with hybrid precoding, accurate channel state information (CSI) estimation is indispensable to achieve better system performance. Due to the spatial sparsity in mmWave channel, traditional channel model based on rich scattering is not practical, while the mmWave channel with a limited number of scattering path can be modeled as a parametric form in terms of the path angles of arrival/departure (AoAs/AoDs) and the corresponding path gains. As a result, the mmWave channel estimation problem can be solved by estimating the path direction (AoAs/AoDs) and path gains instead of estimating the MIMO channel matrix [7, 8].

The construction of orthogonal training sequence of a massive MIMO system is analyzed; by constructing a well orthogonal pilot sequence, channel estimation and interference cancelation can be carried out by using autocorrelation and cross-correlation. Although the orthogonal pilot sequence has good channel estimation performance, since the pilot overhead increases with the number of antennas in the massive MIMO system, the orthogonality of the pilot cannot be satisfied due to the limited time-frequency resources. Therefore, the non- 
orthogonal pilot has become a research hotspot. In multi-cell large-scale MIMO systems, when the training time slot is small enough, the proposed non-orthogonal pilot can obtain the effect of approximating the orthogonal pilot, while the orthogonal pilot is unachievable when the training time slot is small enough [9]. Nonorthogonal pilot is applied to solve the collision detection capacity problem in large-scale connection scenarios, and the channel estimation performance is improved [10]. Li proposed that when the pilot signal is sufficiently long, the Procrustes criterion can be used to reconstruct mutually orthogonal pilot signals; but when the signal is not long enough, the optimal pilot sequence can be reconstructed by the Procrustes criterion and additional block matrix method [11].

In addition to the study of the non-orthogonal pilot, compressed sensing has been extensively studied for massive MIMO system estimation. The application of this theory benefits from the sparsity of the impulse response of the wireless communication system in the delay domain. Research shows that the traditional method of linearly reconstructing channel state based on training sequences is suitable for multipath channel environments with a large number of paths. The physical environment and simulation analysis results show that the wireless channel in many actual situations presents a sparse multipath structure, which can be solved by the method of compressed sensing. In [12], a distributed compressed sensing method is used to solve the sparse channel estimation problem of the MIMOOFDM system. Channel estimation performance and system effectiveness are improved by optimizing pilot settings and using compressed sensing. The channel estimation and precoding problems in mmWave communication systems are resolved in mmWave communication with the idea of compression sensing [13]. In addition to the typical channel sparsity, mmWave communication also has the problem of angle spread, which presents a low-rank structure. In summary, the actual wireless channel presents sparsity or approximate sparsity, for the future mmWave communication systems, the characteristics of sparsity can be even directly used to complete the design of the signal processing algorithm.

The current measurement matrix is nearly all based on the Gaussian random matrix. However, compared with the Gaussian random matrix, the Toeplitz structure measurement matrix has lower generation complexity under the premise of satisfying the finite isometric characteristics of the compressed sensing theory [14]. In addition, in terms of channel estimation performance, the Toeplitz structure sensing matrix proposed in this paper can achieve nearly the same effect as the Gaussian random matrix.
In this paper, we adopt the measurement matrix based on CC-S to implement the channel estimation in a massive MIMO system. Specifically, the main contributions of this paper are as follows:

a) Based on the recursive method, we propose the construction algorithm of CC-S.

b) We break through the conventional measurement matrix based on the Gaussian random matrix and propose the idea of using the Toeplitz structure measurement matrix based on CC-S.

c) Based on the classical massive MIMO scheme, we propose the new massive MIMO system with hybrid precoding. In this way, the complexity can be reduced.

The remainder of this paper is listed as follows. Section 2 describes the measurement matrix construction method based on CC-S. The next section describes the massive MIMO channel estimation algorithm based on CC-S. Section 4 analyzes the performance of the Gaussian random matrix and CC-S. And Section 6 concludes the work.

\section{Methods}

The implementation framework of compressed sensing theory includes signal sparse representation, sensing matrix design, and recovery algorithm design. In a massive MIMO system based on compressed sensing, the design of the measurement matrix is important. Therefore, a new deterministic Toeplitz structure sensing matrix based on the characteristics of CC-S is developed. At the same time, it is proved by theoretical analysis that CC-S satisfies the Spark characteristics. In addition, in terms of channel estimation performance, the orthogonal matching pursuit (OMP) algorithm using CC-S as a measurement matrix is developed. The simulation results show that the Toeplitz structure sensing matrix based on CC-S proposed in this paper can achieve nearly the same effect as the Gaussian random matrix.

\section{Measurement matrix construction method based on CC-S}

\subsection{A construction method of CC-S sets}

Given two length-N complex-valued sequences $\boldsymbol{a}$ (or $\left\{a_{t}\right\}$, i.e., $\left.a_{t}=\left\{a_{0}, a_{1}, \cdots, a_{N-1}\right\}\right)$ and $b$ (or $\left\{b_{t}\right\}$ ), their aperiodic correlation function of positive time shift $\tau$ is defined as:

$$
\psi\left(a_{t}, b_{t} ; \tau\right)=\left\{\begin{array}{cc}
\sum_{t=0}^{N-1-\tau} a_{t}\left(b_{t+\tau}\right)^{*}, & 0 \leq \tau \leq(N-1) \\
0 & \tau \geq N
\end{array}\right.
$$

In this paper, we only consider positive delay for simplicity, without loss of generality, when the negative delay is $\tau<0$, we have: 


$$
\psi\left(a_{t}, b_{t} ; \tau\right)=\left\{\begin{array}{cc}
\sum_{t=0}^{N-1+\tau} a_{t-\tau}\left(b_{t}\right)^{*}, & (1-N) \leq \tau<0 \\
0 & \tau \leq-N
\end{array}\right.
$$

When $a \neq b$, the above is called aperiodic crosscorrelation function (ACCF); otherwise, it is called aperiodic auto-correlation function (AACF). For simplicity, we denote $\psi(a ; \tau)$ to represent the AACF of $\boldsymbol{a}$

Definition 1:.A pair of codes $(\boldsymbol{a}, \boldsymbol{b})$ is said to be a pair of complementary sequence if they satisfy:

$$
\psi(a ; \tau)+\psi(b ; \tau)=C \delta(\tau)
$$

where $C$ is a positive constant and whose value is the sum of the length of $\boldsymbol{a}$ and $b$, and $\delta(\tau)$ is the Kronecker delta function.

$C(K, M, N)$ is a family of CC-S and is named CC-S sets, which contains $K C C$-S with each size is $M \times N$ and denoted as $C^{(k)}, k \in\{0,1, \cdots, K-1\}$. For each CC-S set $C^{(k)}, M$ element sequences $c_{m}^{k}$ with the same code length $\mathrm{N}$ are contained, $m \in\{0,1, \cdots, M-1\}$. Hence, $C^{(k)}$ can be unfolded as a $M \times N$ matrix (Fig. 1).

$$
C^{(k)}=\left[\begin{array}{c}
c_{0}^{(k)} \\
c_{1}^{(k)} \\
\vdots \\
c_{M-1}^{(k)}
\end{array}\right]=\left[\begin{array}{cccc}
c_{0,0}^{(k)} & c_{0,1}^{(k)} & \cdots & c_{0, N-1}^{(k)} \\
c_{1,0}^{(k)} & c_{1,1}^{(k)} & \cdots & c_{1, N-1}^{(k)} \\
\vdots & \vdots & \ddots & \vdots \\
c_{M-1,0}^{(k)} & c_{M-1,1}^{(k)} & \cdots & c_{M-1, N-1}^{(k)}
\end{array}\right]
$$

where $c_{m, n}^{(k)}$ is the specific CC-S with bipolar value and $c_{m, n}^{(k)} \in\{1,-1\}$, while $m \in\{0,1, \cdots, M-1\}, n \in\{0,1, \cdots, N-$ $1\}$, and $k \in\{0,1, \cdots, K-1\}$.

The correlation properties of CC-S are characterized by the complementary aperiodic correlation function, which

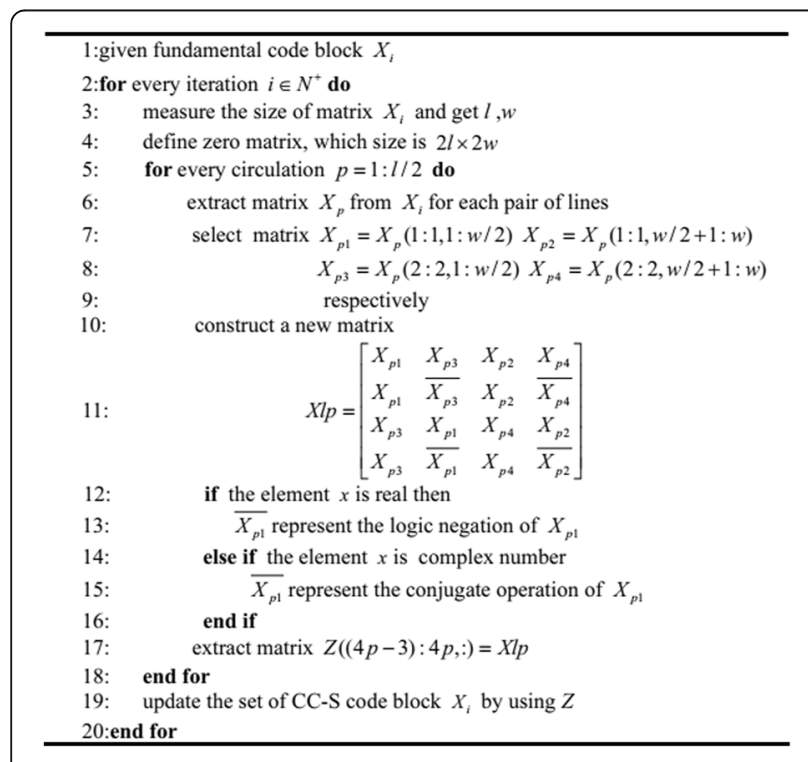

Fig. 1 Construction method of CC-S is calculated as the sum of the aperiodic correlation functions of all element codes with the same delay $\tau$.

\subsection{Design standard of measurement matrix}

We have known that in compressed sensing, the signal is first represented as a sparse form. A signal $\mathbf{x}$ of dimension $N$ can be represented in the form of another vector under a set of orthogonal basis vectors $\left\{\mathbf{a}_{k}\right\}_{k=1}^{N}$, and the vector $K$ is sparse. After signal sparseness, the measurement matrix must be designed. By using the observation matrix for data processing, we can get a lowdimensional observation vector $\mathbf{y}$ with dimension $M(M \ll N)$ :

$$
\mathbf{y}=\boldsymbol{\Phi} \mathbf{x}=\boldsymbol{\Phi A S}=\mathbf{\Theta S}
$$

where $\Phi$ represents the measurement matrix with dimension $M \times N, \boldsymbol{\Theta}=\boldsymbol{\Phi} \mathbf{A}$ is the observation matrix, and $\mathrm{y}$ is the observed low-dimensional vector.

In (4), since the dimension of the $\Theta$ matrix is measured as $M \times N$, when we get the result of $\mathbf{x}$, the above equation is an underdetermined equation, and the number of equations is much smaller than the number of unknowns, it is very difficult to solve directly. If the dimension of the observed sample $\mathbf{y}$ and the sparsity of the original signal in the transform domain can satisfy $M>K$, the observation matrix $\Theta$ satisfies certain conditions, and the process of sparse vector restoration can be regarded as solving the optimal $\ell_{0}$-norm problem by the measured value $\mathbf{y}$, which can be expressed as:

$$
\hat{\mathbf{S}}=\operatorname{argmin}\|\mathbf{S}\|_{0} \quad \text { s.t. } \Theta \mathbf{S}=\mathbf{y}
$$

In the case where the signal $\mathbf{S}$ is obtained, if the position of the $K$ non-zero elements in $\mathbf{S}$ is known, the equation of the original $M \times N$ can be converted into the equation of $M \times K$ to solve. If the value of $K$ non-zero elements in $\mathbf{S}$ can be obtained, the original signal $\mathbf{x}$ can be recovered by the following formula:

$$
\mathbf{x}=\sum_{k=1}^{N} \mathbf{a}_{k} s_{k}=\mathbf{A S}
$$

In this process, the solution with the coefficient $M \times K$ equation is not unique, but the entire compression recovery process needs to be guaranteed to be unique, so the requirements for $\Theta$ are imposed. The literature [15] proposes that $\Theta$ needs to meet the restricted isometry property (RIP) condition to complete the reconstruction of the sparse signal.

Definition 2: RIP condition: For any vector set $\mathbf{c} \in$ $\mathbb{R}^{|T|}$ and constant $\delta_{K} \in(0,1)$, if the following formula holds that $\Theta$ is said to satisfy the RIP condition: 


$$
\left(1-\delta_{K}\right)\|\mathbf{c}\|_{2}^{2} \leq\left\|\Theta_{T} \mathbf{c}\right\|_{2}^{2} \leq\left(1+\delta_{K}\right)\|\mathbf{c}\|_{2}^{2}
$$

where $T \subset\{1, \cdots, N\}, \quad|T| \leq K$, and $\Theta_{T}$ are submatrices of dimension $K \times|T|$ formed by index $T$ indication of the measurement matrix $\Theta$.

In general, for a $K$-sparse signal $\mathbf{S}$ (the position of $K$ non-zero elements is unknown), the sufficient condition for reconstructing the signal $\mathbf{S}$ from $\mathbf{y}$ by Eq. (5) is that the arbitrary vector set $\mathbf{c}$ and the constant $\delta_{2 K} \in(0,1)$ satisfy the following formula, that is, satisfy the $2 K$ order RIP condition:

$$
\left(1-\delta_{2 K}\right)\|\mathbf{c}\|_{2}^{2} \leq\left\|\Theta_{T} \mathbf{c}\right\|_{2}^{2} \leq\left(1+\delta_{2 K}\right)\|\mathbf{c}\|_{2}^{2}
$$

where $T \subset\{1, \cdots, N\},|T| \leq 2 K$.

In the construction of the measurement matrix, the $2 K$ order RIP conditions are more difficult to satisfy. The literature [16] pointed out that the measurement matrix satisfying the RIP condition is equivalent to the uncorrelation between the observation matrix $\Phi$ and the orthogonal basis $\mathbf{A}$. In other words, it is required that row $\phi_{j}$ in the matrix $\boldsymbol{\Phi}$ cannot be sparsely represented by column $\mathbf{a}_{i}$ in $\mathbf{A}$, and column $\mathbf{a}_{i}$ in $\mathbf{A}$ cannot be sparsely represented by the $\phi_{j}$ in $\boldsymbol{\Phi}$. Since the orthogonal basis $\mathbf{A}$ is fixed, the RIP condition of the measurement matrix $\Phi$ can be realized by designing the observation matrix $\Phi$.

Regarding the design of the observation matrix $\Phi$, when the observation matrix $\boldsymbol{\Phi}$ is a Gaussian random matrix (the dimension is $M \times N$, and the inner element value satisfies the independent normal distribution of $N(0,1 / N))$, the measurement matrix $\boldsymbol{\Phi}$ can satisfy the RIP condition with a large probability.

In addition, the design types of the observation matrix $\boldsymbol{\Phi}$ include a uniform sphere measurement matrix, a local Fourier matrix, a local Hadamard measurement matrix, and a Toeplitz matrix. When the column of the measurement matrix $\boldsymbol{\Phi}$ presents independent identically distributed (i.i.d.) on the ball $S^{n-1}$, and when the number of measurements $M \geq \mathcal{O}(K \ln (N))$, the probability of successfully reconstructing the signal is large, and the measurement matrix $\Phi$ is referred to as a uniform sphere measurement matrix. The local Fourier matrix is a random selection of $M$ rows from the $N \times N$ Fourier matrix, and then the regularization of the new matrix is obtained. The advantage is that it can be realized by the fast Fourier transform; the disadvantage is that whether it can satisfy the correlation depends on the nature of the sparse signal. The local Hadamard measurement matrix is obtained by randomly selecting $M$ rows from the $N \times N$ Fourier matrix.

In the content of research on compressed sensing, a Gaussian random matrix is generally used. However, there are application defects in the Gaussian random matrix, because the random characteristics, matrix generation, and storage process require a large amount of storage space and computational complexity. Based on this, a new Toeplitz observation matrix is proposed in this paper. By applying the excellent correlation properties of complete complementary sequences, a Toeplitz observation matrix based on CC-S is designed. When applied with compressed sensing channel estimation, it can obtain similar channel estimation performance from the traditional Gaussian random matrix, but the measurement matrix implementation is less complex.

\section{Massive MIMO channel estimation based on CC- $S$}

\subsection{System model}

Figure 2 shows the structure of mmWave massive MIMO system with hybrid precoding. The base station (BS) with $N_{\mathrm{BS}}$ antennas and $N_{\mathrm{RF}}^{\mathrm{BS}}$ chains communicates with a single mobile station (MS)-equipped $N_{\mathrm{MS}}$ antennas and $N_{\mathrm{RF}}^{\mathrm{MS}}$ RF chains. The BS and MS communicate via $N_{\mathrm{S}}$ streams and satisfy $N_{\mathrm{S}} \leq N_{\mathrm{RF}}^{\mathrm{BS}} \leq N_{\mathrm{BS}}$ and $N_{\mathrm{S}} \leq N_{\mathrm{RF}}^{\mathrm{MS}} \leq$ $N_{\text {MS }}$. In this paper, we focus on the single-cell downlink transmission. Baseband precoder $F_{\mathrm{BB}} \in \mathbb{C}^{N_{\mathrm{RF}}^{\mathrm{BS}} \times N_{\mathrm{S}}}$ is followed by RF precoder $F_{\mathrm{RF}} \in \mathbb{C}^{N_{\mathrm{BS}} \times N_{\mathrm{RF}}^{\mathrm{BS}}}$, while the hybrid precoder at the transmitter is defined as $F=F_{\mathrm{BB}} \times F_{\mathrm{RF}}$ $\in \mathbb{C}^{N_{\mathrm{BS}} \times N_{\mathrm{S}}}$. The discrete-time transmitted signal is:

$$
\mathbf{x}=\mathbf{F s}
$$

We consider a narrowband block fading channel model in which the received signal is:

$$
\mathbf{r}=\mathbf{H F s}+\mathbf{n}
$$

where $s$ is the $N_{\mathrm{S}} \times 1$ vector of the transmitted symbol. $\mathrm{H}$ $\in \mathbb{C}^{N_{\mathrm{MS}} \times N_{\mathrm{BS}}}$ represents the mmWave channel matrix between the BS and MS, and $\mathbf{n} \in \mathbb{C}^{N_{\mathrm{MS}} \times 1}$ is the complex additive white Gaussian noise (AWGN) corresponding to the MS and follows the distribution $\mathcal{C N}\left(0, \sigma_{n}^{2} I\right)$. At the MS, the combiner is defined as $W=W_{\mathrm{RF}} \times W_{\mathrm{BB}} \in$ $\mathbb{C}^{N_{\mathrm{S}} \times N_{\mathrm{MS}}}$, where $W_{\mathrm{RF}} \in \mathbb{C}^{N_{\mathrm{MS}} \times N_{\mathrm{RF}}^{\mathrm{MS}}}$ is the RF combiner and $W_{\mathrm{BB}} \in \mathbb{C}^{N_{\mathrm{RF}}^{\mathrm{MS}} \times e N_{\mathrm{S}}}$ is the baseband combiner. After being processed at the receiver, the received signal is:

$$
\mathbf{y}=\mathbf{W}^{\mathrm{H}} \mathbf{H F} \mathbf{s}+\mathbf{W}^{\mathrm{H}} \mathbf{n}
$$

In this paper, a uniform rectangular array (URA) 2D geometry model is considered, where $M$ and $N$ are the element number of antennas in the $x-y$ plane, and the space of adjacent antenna is $\lambda / 2$, as illustrated in Fig. 3.

Since the mmWave channel model has limited scattering, we adopt a geometric channel with $L$ scatters. Each scatter assumed to contribute one single propagation path between BS and MS. While the channel model can be written as: 


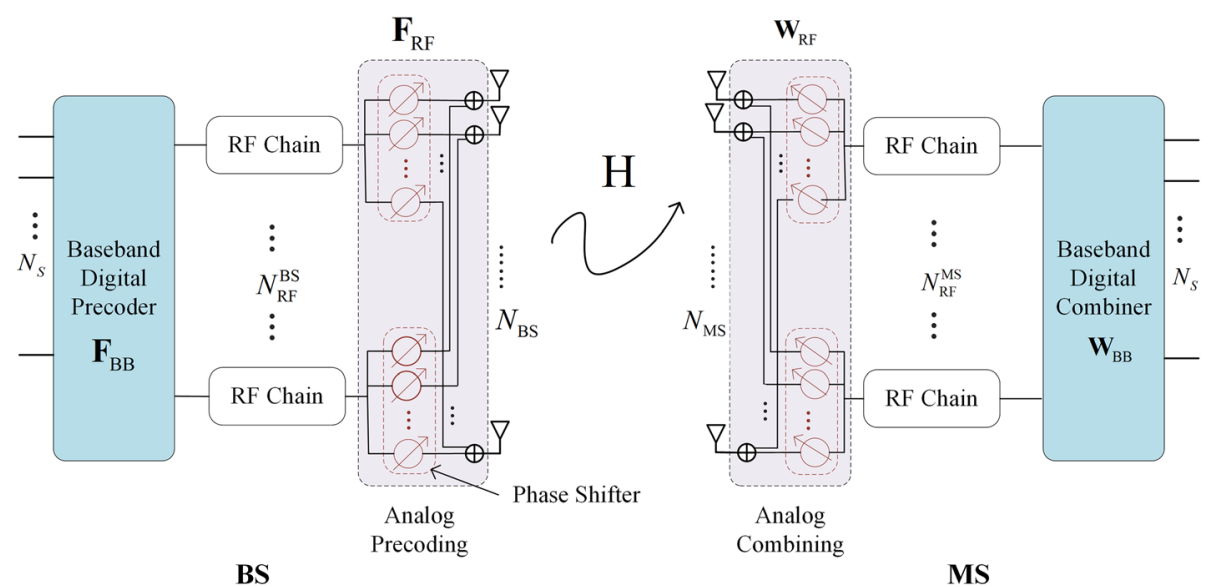

Fig. 2 Illustration of a hybrid analog/digital precoding and combing structure

$$
H=\sqrt{\frac{N_{\mathrm{BS}} N_{\mathrm{MS}}}{\rho}} \sum_{\ell=1}^{L} g_{\ell} A_{R}\left(\theta_{\ell, R}, \phi_{\ell, R}\right) A_{T}^{H}\left(\theta_{\ell, T}, \phi_{\ell, T}\right)
$$

where $\rho$ denotes the average path loss between BS and MS, and $g_{\ell}$ is the complex gain of the $\ell$ th path assumed to be Rayleigh distributed $g_{\ell^{\sim \mathcal{N}}}(0, \bar{P})$ with $\bar{P}$ the average power gain. The variable $\phi_{\ell}, T, \phi_{\ell}$, ${ }_{R} \in[0, \pi]$ denotes the elevation angles, and $\theta_{\ell}, T, \theta_{k}$, ${ }_{R} \in[0,2 \pi]$ denotes the azimuth angles corresponding to the $\ell$ th path. $A_{T}\left(\theta_{\ell, T}, \phi_{\ell, T}\right)$ and $A_{R}\left(\theta_{\ell, R}, \phi_{\ell, R}\right)$ denote the array response at BS and MS, respectively.

We adopt URAs; hence, the $A_{T}\left(\theta_{\ell, T}, \phi_{\ell, T}\right)$ and $A_{R}\left(\theta_{\ell}\right.$, $\left.{ }_{R}, \phi_{\ell, R}\right)$ can be expressed as:

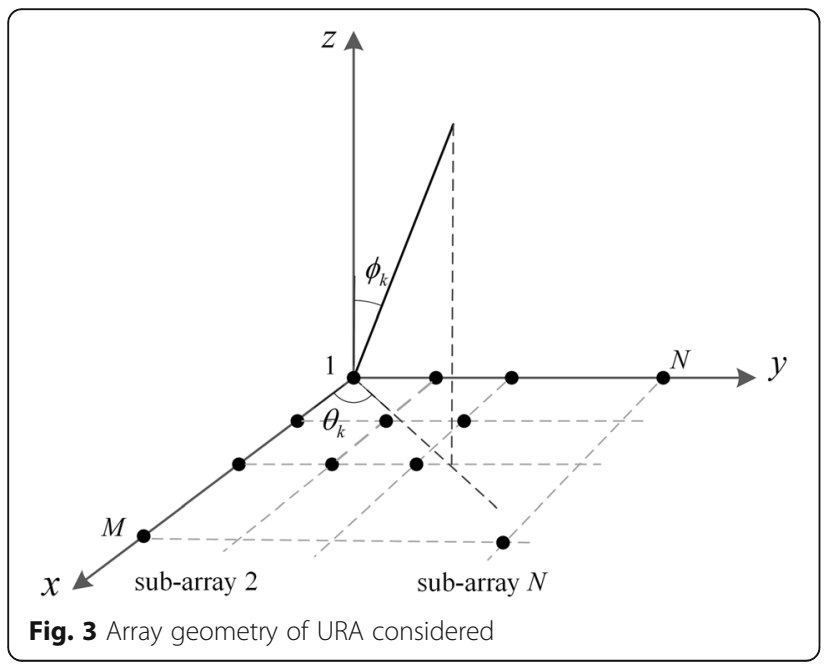

$$
\begin{aligned}
& \mathbf{A}_{T}\left(\theta_{\ell, T}, \phi_{\ell, T}\right)=\mathbf{a}_{y, T}\left(\theta_{\ell, T}, \phi_{\ell, T}\right) o \mathbf{a}_{x, T}\left(\theta_{\ell, T}, \phi_{\ell, T}\right) \\
& \mathbf{A}_{R}\left(\theta_{\ell, R}, \phi_{\ell, R}\right)=\mathbf{a}_{y, R}\left(\theta_{\ell, R}, \phi_{\ell, R}\right) o \mathbf{a}_{x, R}\left(\theta_{\ell, R}, \phi_{\ell, R}\right)
\end{aligned}
$$

where

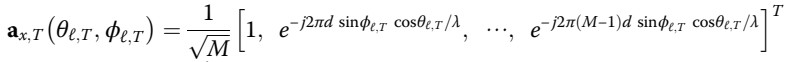

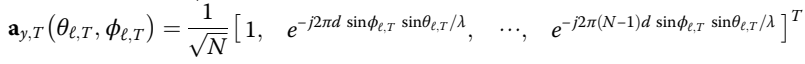

They denote the sub-array response in the vertical and horizontal directions at the BS. Similarly,

$\mathbf{a}_{x, R}\left(\theta_{\ell, R}, \phi_{\ell, R}\right)=\frac{1}{\sqrt{M}}\left[\begin{array}{llll}1, & e^{-j 2 \pi d \sin \phi_{\ell, R} \cos \theta_{\ell, R} / \lambda}, & \cdots, & e^{-j 2 \pi(M-1) d \sin \phi_{\ell, R} \cos \theta_{\ell, R} / \lambda}\end{array}\right]^{T}$
$\mathbf{a}_{y, R}\left(\theta_{\ell, R}, \phi_{\ell, R}\right)=\frac{1}{\sqrt{N}}\left[\begin{array}{llll}1, & e^{-j 2 \pi d \sin \phi_{\ell, R} \sin \theta_{\ell, R} / \lambda}, & \cdots, & e^{-j 2 \pi(N-1) d \sin \phi_{\ell, R} \sin \theta_{\ell, R} / \lambda}\end{array}\right]^{T}$

They denote the sub-array response in the vertical and horizontal directions at the MS. o denotes the KhatriRao product. The channel in (12) is written in a more compact form:

$$
\mathbf{H}=\mathbf{A}_{\mathbf{R}} \Lambda \mathbf{A}_{\mathrm{T}}^{\mathrm{H}}
$$

where $\Lambda \in \mathbb{C}^{L \times L}$ is the channel complex gain matrix with elements $\sqrt{\frac{N_{\mathrm{BS}} N_{\mathrm{MS}}}{\rho}} \operatorname{diag}\left[g_{1}, \cdots, g_{L}\right]$.

$\mathbf{A}_{T}=\left[\mathbf{a}_{y, T}\left(\theta_{1, T}, \phi_{1, T}\right) \otimes \mathbf{a}_{x, T}\left(\theta_{1, T}, \phi_{1, T}\right), \cdots, \mathbf{a}_{y, T}\left(\theta_{\ell, T}, \phi_{\ell, T}\right) \otimes \mathbf{a}_{x, T}\left(\theta_{\ell, T}, \phi_{\ell, T}\right)\right] \in C^{N_{\mathrm{BS}} \times L}$ $\mathbf{A}_{R}=\left[\mathbf{a}_{y, R}\left(\theta_{1, R}, \phi_{1, R}\right) \otimes \mathbf{a}_{x, R}\left(\theta_{1, R}, \phi_{1, R}\right), \cdots, \mathbf{a}_{y, R}\left(\theta_{\ell, R}, \phi_{\ell, R}\right) \otimes \mathbf{a}_{x, R}\left(\theta_{\ell, R}, \phi_{\ell, R}\right)\right] \in C^{N_{M S} \times L}$

where $\otimes$ denotes the Kronecker product. The derivation of Eq. (17) is shown in Appendix. 


\subsection{Channel estimation algorithm based on CC-S}

The mmWave channels have obvious sparsity performance in the angular domain when the number of nonline-of-sight is small. We can transform the frequency domain into a sparse domain in order to apply the compressed sensing technology through the following DFT transform matrix:

$$
\mathbf{H}^{s}=\mathbf{D}_{\mathrm{BS}}^{*} \mathrm{HD} \mathbf{D E}_{\mathrm{UE}}
$$

where $\mathbf{D}_{\mathrm{BS}}$ and $\mathbf{D}_{\mathrm{UE}}$ are the DFT matrices at the base station and user end, respectively. $\mathbf{H}^{s}$ denotes the sparse channel matrix in the angle domain. We can obtain the vector $\mathbf{h}$ by vectorizing $\mathbf{H}$ :

$$
\begin{aligned}
& \mathbf{h}^{s}=\operatorname{vect}\left(\mathbf{H}^{s}\right)=\left[\left(\mathbf{D}_{\mathrm{UE}}^{*}\right)^{T} \otimes \mathbf{D}_{\mathrm{BS}}\right] \mathbf{h} \\
& \mathbf{h}=\operatorname{vect}(\mathbf{H})
\end{aligned}
$$

We can further obtain the received signal as:

$$
\begin{aligned}
\mathbf{y} & =\mathbf{W}^{H} \mathbf{D}_{\mathrm{BS}} \mathbf{H}^{s} \mathbf{D}_{\mathrm{UE}}^{*} \mathbf{F s}+\mathbf{W}^{\mathbf{H}} \mathbf{n} \\
& =\left(\mathbf{D}_{\mathrm{UE}}^{*} \mathbf{F s}\right)^{T} \otimes\left(\mathbf{W}^{H} \mathbf{D}_{\mathrm{BS}}\right) \mathbf{h}^{s}+\tilde{\mathbf{n}} \\
& =\mathbf{\Phi} \mathbf{h}^{s}+\tilde{\mathbf{n}}
\end{aligned}
$$

Our purpose is to accurately estimate the channel information from (21). The sparsity of the massive MIMO channels helps us to use the compressed sensing technology to estimate the channel state with reduced pilot overhead. According to [17], channel $\mathrm{H}$ share the same $\mathrm{AOA}$ and AOD; $\mathbf{H}^{s}$ has the structured sparsity. Specifically, we can use a standard DCS algorithm to estimate the AOA, AOD, and the channel gain information. The aim of this paper is to verify the performance of Toeplitz-type observation matrix based on CC-S.

We will compare the performance difference between the Toeplitz structure measurement matrix based on the complementary code and the Gaussian random matrix under the compressed sensing framework. In this section, the sparse representation of the signal uses a discrete Fourier basis. The sensing matrix is a Toeplitz structural measurement matrix based on complementary sequences and a Gaussian random matrix. If there is no special explanation, use $\Phi \in \mathbb{R}^{64 \times 256}$; the signal recovery algorithm adopts the OMP algorithm. The following is a brief description of the OMP core algorithm steps:

1. Input: measurement matrix $\boldsymbol{\Phi}$, observation vectory, sparsity $K$

2. Output: the coefficient of $\mathbf{x}$ approximates the vector $\hat{\mathbf{x}}$

3. Initialization: $\mathbf{r}_{0}=\mathbf{y}$, index set $\Gamma_{0}=, t=1$

4. Loop through steps 1-5:

(a) Step 1: Find the position $i$ corresponding to the maximum value of residual $\mathbf{y}_{r}$ and column $\boldsymbol{\phi}_{i}$ product in the observation matrix isi $i_{t}=\arg$ $\max _{j=1 \ldots N}\left|\quad \mathbf{r}_{t=1}, \boldsymbol{\phi}_{j} \quad\right|$.

(b) Step 2: Update the index set $\Gamma_{t}=\Gamma_{t} \cup\left\{i_{t}\right\}$ and record the set of reconstructed atoms $\boldsymbol{\Phi}_{t}=$ [ $\left.\boldsymbol{\Phi}_{t-1}, \boldsymbol{\Phi}_{i_{t}}\right]$ in the sensing matrix.

(c) Step 3: Calculate $\hat{\mathbf{x}}_{t}=\operatorname{argmin}\left\|\mathbf{y}-\boldsymbol{\Phi}_{t} \hat{\mathbf{x}}\right\|_{2}$.

(d) Step 4: Update the residual $\mathbf{r}_{t}=\mathbf{y}-\boldsymbol{\Phi}_{t} \hat{\mathbf{x}}$ and $t=$ $t+1$

(e) Step 5: Determine if $t>K$ is satisfied. If it is satisfied, stop iteration; if not, go to step 1 .

\subsection{Compressed sensing channel estimation under a new observation matrix}

In compression sensing, the design of the observation matrix and the signal recovery algorithm are the focus of research. The design of the observation matrix needs to be as practical as possible while meeting the signal recovery requirements. According to [18], the elements of the measurement matrix should follow an i.i.d. Gaussian distribution in order to get the better performance for spare domain signal recovery. Since the Gaussian random matrix is almost irrelevant to any sparse signal, the RIP condition can be satisfied with a large probability. However, there are application defects in the Gaussian random matrix. Because of the random characteristics, matrix generation and storage processes require a large amount of storage space and computational complexity.

Bajwa and Haupt et al. proposed the Toeplitz structural observation matrix and the cyclic matrix. The Toeplitz structural measurement matrix is an ideal choice in many applications, and it mainly includes the following three reasons:

(1) The Gaussian random matrix (i.i.d. measurement matrix) needs to generate $\mathcal{O}(k n)$ independent random variables, which is not desirable when the vector dimension is high. In contrast, the Toeplitztype measurement matrix only needs to generate $\mathcal{O}$ (n)independent random variables.

(2) During the signal processing process, the i.i.d. measurement matrix needs to be operated $\mathcal{O}(k n)$ times when it is "multiplied," which results in a long time for the acquisition and reconstruction of largedimensional data. The Toeplitz-type measurement matrix "multiplication" can be realized by fast Fourier transform with a complexity of $\mathcal{O}\left(n \log _{2}(n)\right)$.

(3) The Toeplitz-type measurement matrix can be applied to specific fields, such as it matches the linear time-varying system, but the i.i.d. measurement matrix does not apply to this kind of scene.

This section presents a Toeplitz structural measurement matrix based on complementary sequences. Since 
the complementary sequences have excellent crosscorrelation properties, the non-correlation conditions of the measurement matrix can be satisfied. At the same time, the Spark characteristics of the matrix are analyzed, and it is proved that it can meet the requirements of the measurement matrix. Subsequently, the Toeplitztype measurement matrix is applied to the scene of compressed sensing MIMO channel estimation. The experimental results show that the sensing matrix proposed in this paper can achieve a similar signal recovery effect with the Gaussian random matrix. Thanks to its Toeplitz structure, the new sensing matrix proposed in this section is more practical.

\subsection{Design of Toeplitz structure measurement matrix based on CCs}

The measurement matrix needs to meet the RIP conditions in order to recover the original signal with a high probability. In the measurement matrix design, the Spark (minimum linear correlation columns) value of the measurement matrix is also the focus of attention. The definition of the Spark value is given below:

Definition 3: The Spark value of the measurement matrix $\Phi$ is defined as:

$$
\operatorname{Sp}(\boldsymbol{\Phi})=\min \left\{\|\boldsymbol{\omega}\|_{0}: \boldsymbol{\omega} \in \boldsymbol{\Phi}_{\text {Nullsp }_{R}}{ }^{*}\right\}
$$

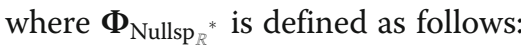

$$
\boldsymbol{\Phi}_{\text {Nullsp }_{R}^{*}}=\left\{\boldsymbol{\omega} \in \mathbb{R}^{N}: \boldsymbol{\Phi} \boldsymbol{\omega}=0, \boldsymbol{\omega} \neq 0\right\}
$$

The literature [19] proves that when the Spark value of the measurement matrix $\Phi$ satisfies the following conditions, the signal estimation value can be obtained by solving the minimum $\ell_{0}$-norm optimization problem of (5).

$$
\operatorname{Sp}(\Phi) \geq 2 K
$$

The traditional i.i.d. Toeplitz matrix has the following form:

$$
\boldsymbol{\Phi}=\left[\begin{array}{ccccc}
a_{N} & a_{N-1} & \cdots & a_{2} & a_{1} \\
a_{N+1} & a_{N} & \cdots & a_{3} & a_{2} \\
\vdots & \vdots & \ddots & \ddots & \vdots \\
a_{N+M-1} & a_{N+M-2} & \cdots & \cdots & a_{M}
\end{array}\right]
$$

The inner element $\left\{a_{i}\right\}_{i=1}^{N+M-1}$ obeys the i.i.d distribution with probability $P(a)$ and the column vector normalization at the same time (the row vector is also normalized). Taking the first $M$ lines constitutes the form of the above-mentioned partial Toeplitz matrix.

The i.i.d. Toeplitz matrix whose element selection obeys the i.i.d distribution with a probability $P(a)$. Due to the element distribution problem, the atom (the column vector of the matrix) in the i.i.d. Toeplitz matrix can satisfy the correlation requirement. Unlike the traditional i.i.d. Toeplitz matrix, the elements of the Toeplitz structure measurement matrix based on complementary sequences proposed in this section are deterministic.

The form of the Toeplitz structure measurement matrix based on complementary sequences is given below:

$$
\boldsymbol{\Phi}=\left[\begin{array}{cccccccccc}
a_{0} & a_{1} & \cdots & a_{N-2} & a_{N-1} & b_{0} & b_{1} & \cdots & b_{N-2} & b_{N-1} \\
a_{1} & a_{2} & \cdots & a_{N-1} & a_{0} & b_{1} & b_{2} & \cdots & b_{N-1} & b_{0} \\
\vdots & \vdots & \ddots & \vdots & \vdots & \vdots & \vdots & \ddots & \vdots & \vdots \\
a_{N-1} & a_{0} & \cdots & a_{N-3} & a_{N-2} & b_{N-1} & b_{0} & \cdots & b_{N-3} & b_{N-2}
\end{array}\right]
$$

where $\left\{a_{i}\right\}_{i=0}^{N-1}$ and $\left\{b_{i}\right\}_{i=0}^{N-1}$ are a pair of complementary sequences of length $N$, and each element takes a value in the binary domain $\{1,-1\}$. As shown in (26), the directly generated Toeplitz structure based on the complementary sequence has a dimension of $N \times 2 N$, and the sampling rate is 0.5 .

Using the complementary sequences, the Toeplitz structural measurement matrix can be constructed by the cyclic structure. When we apply the Toeplitz structural measurement matrix to the compressed sensing signal recovery, we can obtain the performance similar to the Gaussian random matrix. We will theoretically analyze the Toeplitz structural measurement matrix based on complementary codes and analyze its Spark characteristics to demonstrate its feasibility as a measurement matrix.

\subsection{Analysis of Spark characteristics of new Toeplitz matrix}

When the sparseness of the signal satisfies $K \leq \mathrm{Sp}(\Phi) / 2$, the signal estimate can be obtained by solving the minimum $\ell_{0}$-norm optimization problem of (5). However, it is difficult to calculate the value of matrix Spark. By converting the Spark value into the calculated measurement matrix correlation value, it is easier to judge whether the matrix meets the requirements of the compressed sensing measurement matrix. Specifically, the range of the matrix Spark value can be determined by calculating the correlation of the measurement matrix. The definition of the correlation of the measurement matrix is given below:

Definition 4: For matrix $\boldsymbol{\Phi}=\left(\boldsymbol{\phi}_{1}, \boldsymbol{\phi}_{2}, \cdots \boldsymbol{\phi}_{N}\right) \in \mathbb{R}^{M \times N}$, the correlation $\mu(\Phi)$ is:

$$
\mu(\boldsymbol{\Phi})=\max _{1 \leq p \neq q \leq N} \frac{\left|\left\langle\boldsymbol{\Phi}_{p}, \boldsymbol{\Phi}_{q}\right\rangle\right|}{\left\|\boldsymbol{\Phi}_{p}\right\|_{2}\left\|\boldsymbol{\Phi}_{q}\right\|_{2}}
$$

where $\left\langle\boldsymbol{\Phi}_{p}, \boldsymbol{\Phi}_{q}\right\rangle=\boldsymbol{\Phi}_{q}{ }^{T} \boldsymbol{\Phi}_{p}$ represents the vector inner product. 
When the correlation of a matrix is given, the following relationship exists:

$$
\operatorname{Sp}(\boldsymbol{\Phi}) \geq 1+1 / \mu(\Phi)
$$

Then, start with the correlation $\mu(\boldsymbol{\Phi})$ to analyze the Spark properties of the Toeplitz structure measurement matrix based on complementary sequences.

Since both sequences $\left\{a_{i}\right\}_{i=0}^{N-1}$ and $\left\{b_{i}\right\}_{i=0}^{N-1}$ take value on the binary field $\{1,-1\}$, we have:

$$
\begin{aligned}
\left\|\boldsymbol{\Phi}_{p}\right\|_{2}=\left\|\boldsymbol{\Phi}_{q}\right\|_{2}= & \left(\sum_{i=0}^{N-1} a_{i}^{2}\right)^{1 / 2}=\left(\sum_{i=0}^{N-1} b_{i}^{2}\right)^{1 / 2}=N^{1 / 2} \\
& \sum_{i=0}^{N-1} a_{i} a_{i+j}, \quad \boldsymbol{\Phi}_{p}=\left\{a_{i}\right\}, \boldsymbol{\Phi}_{q}=\left\{a_{i+j}\right\}, \quad j \neq 0 \\
7\left\langle\boldsymbol{\phi}_{p}, \boldsymbol{\Phi}_{q}\right\rangle=\boldsymbol{\Phi}_{q}{ }^{T} \boldsymbol{\Phi}_{p}= & \left\{\sum_{i=0}^{N-1} a_{i} b_{i+j}, \quad \boldsymbol{\Phi}_{p}=\left\{a_{i}\right\}, \boldsymbol{\Phi}_{q}=\left\{b_{i+j}\right\},\right. \\
& \sum_{i=0}^{N-1} b_{i} b_{i+j}, \quad \boldsymbol{\Phi}_{p}=\left\{b_{i}\right\}, \boldsymbol{\Phi}_{q}=\left\{b_{i+j}\right\} \quad j \neq 0
\end{aligned}
$$

Bring formula (29) and the formula (30) into formula (27), the correlation value of the matrix can be obtained:

$$
\begin{aligned}
\mu(\boldsymbol{\Phi}) & =\max _{1 \leq p \neq q \leq N} \frac{\left|\left\langle\boldsymbol{\Phi}_{p}, \boldsymbol{\Phi}_{q}\right\rangle\right|}{\left\|\boldsymbol{\Phi}_{p}\right\|_{2}\left\|\boldsymbol{\Phi}_{q}\right\|_{2}} \\
& =\max \left(\frac{1}{N}\left|\sum_{i=0}^{N-1} a_{i} a_{i+j}\right|, \frac{1}{N}\left|\sum_{i=0}^{N-1} a_{i} b_{i+j}\right|, \frac{1}{N}\left|\sum_{i=0}^{N-1} b_{i} b_{i+j}\right|\right)
\end{aligned}
$$

Calculate the above formula to get two values:

$$
\mu(\boldsymbol{\Phi})=\max \{1,0\}
$$

The probability of occurrence of $\{1,0\}$ two values varies greatly:

$$
P\{\mu(\boldsymbol{\Phi})=0\} \gg P\{\mu(\boldsymbol{\Phi})=1\}
$$

From formula (28) and formula (33), the Spark lower bound of the Toeplitz structure measurement matrix of the complementary sequence can be calculated, we have:

$$
\mathrm{Sp}(\boldsymbol{\Phi}) \geq 1+\frac{1}{\mu(\boldsymbol{\Phi})}=\{\infty, 2\}
$$

Definition 5: When the sparsity of the signal satisfies the following formula, the signal estimate can be obtained by solving the minimum $\ell_{0}$-norm optimization problem of (5):

$$
K \leq \operatorname{Sp}(\boldsymbol{\Phi}) / 2
$$

From the above reasoning, $K$ has two values:
When $K=1$, the signal estimate can be obtained by solving the minimum norm optimization problem of (5). When $K>1$, in practice, signal reconstruction can be achieved by increasing the number of measurements.

When $K$ is not limited, but in the actual compression sensing problem, $K$ is a finite constant $(K \ll N)$ compared to the dimension of the original signal vector.

In both cases, the Toeplitz structural measurement matrix based on the complementary code can recover the original signal with high probability. Therefore, through the Spark characteristic analysis, it can be concluded that the Toeplitz structural measurement matrix can be applied to the compressed sensing as the measurement matrix.

\section{Results and discussion}

Simulations are carried out based on the data model and ESPRIT algorithm to investigate channel estimation performance and computational complexity. A $N_{\mathrm{BS}}=N_{\mathrm{MS}}=$ 64 half-wavelength pacing URAs at BS and MS is considered. The sparsity $K=8$. The number of RF chains $N_{\mathrm{RF}}^{\mathrm{MS}}=N_{\mathrm{RF}}^{\mathrm{MS}}=8$. We consider three independent narrowband signals. The directions are generated as $\theta_{\ell}$, $T=\left[10^{\circ} 20^{\circ} 30^{\circ}\right], \phi_{\ell}, T=\left[15^{\circ} 25^{\circ} 35^{\circ}\right]$, and $\theta_{\ell, R}=\left[20^{\circ}\right.$ $\left.30^{\circ} 40^{\circ}\right]$ and $\phi_{\ell, R}=\left[25^{\circ} 35^{\circ} 45^{\circ}\right]$. For each SNR, 1000 Monte Carlo simulations are implemented. The dimension of the measurement matrix is $64 \times 256$. The modulation type is QPSK. In this paper, to measure AoAs/ AoDs estimation performance, the root mean square error (RMSE) is drawn into and is expressed as:

$$
\begin{aligned}
\operatorname{RMSE}_{T} & =\sqrt{\frac{1}{Q L} \sum_{\ell=1}^{L} \sum_{q=1}^{Q}\left[\left(\hat{\phi}_{\ell, \mathrm{T}}-\phi_{\ell, \mathrm{T}}\right)^{2}+\left(\hat{\theta}_{\ell, \mathrm{T}}-\theta_{\ell, \mathrm{T}}\right)^{2}\right]} \\
\operatorname{RMSE}_{\mathrm{R}} & =\sqrt{\frac{1}{Q L} \sum_{\ell=1}^{L} \sum_{q=1}^{Q}\left[\left(\hat{\phi}_{\ell, \mathrm{R}}-\phi_{\ell, \mathrm{R}}\right)^{2}+\left(\hat{\theta}_{\ell, \mathrm{R}}-\theta_{\ell, \mathrm{R}}\right)^{2}\right]}
\end{aligned}
$$

We use normalized mean square error (NMSE) to measure the accuracy of channel estimation, and the NMSE is defined as:

$$
\mathrm{NMSE}=\frac{E\left[\sum_{\ell=1}^{L}\|\mathrm{H}-\hat{\mathrm{H}}\|^{2}\right]}{E\left[\sum_{\ell=1}^{L}\|\mathrm{H}\|^{2}\right]}
$$

In Fig. 4, we compare the RMSE performance of the AOAs and AODs in different targets against SNR of the considered three targets. We can find that the AOAs and AODs can be estimated correctly. In addition, we can see in Fig. 4 that the performance with one target is better than more targets. 


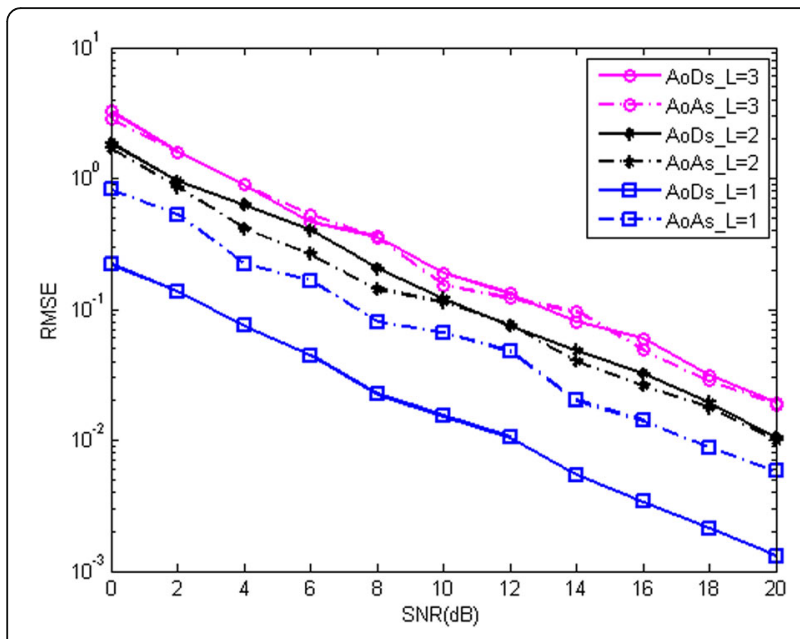

Fig. 4 AoAs and Aods against SNR

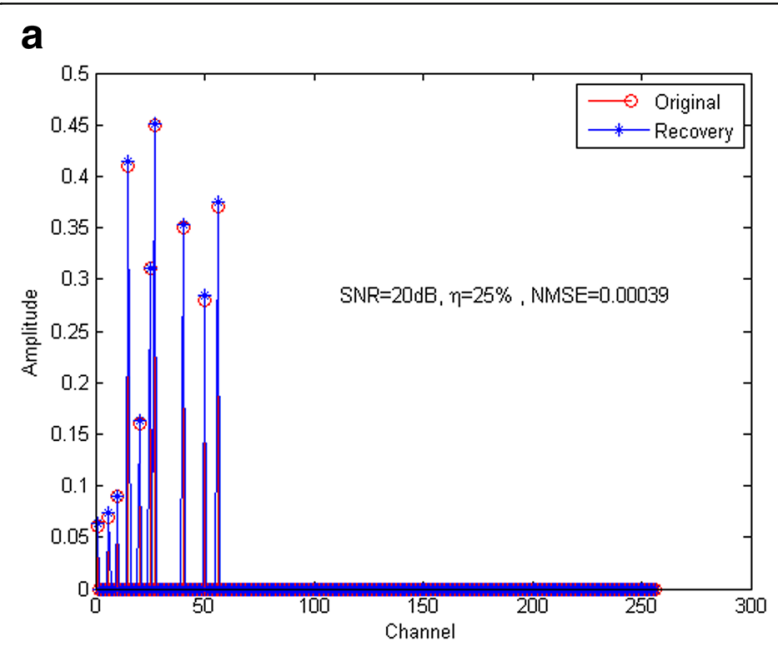

b

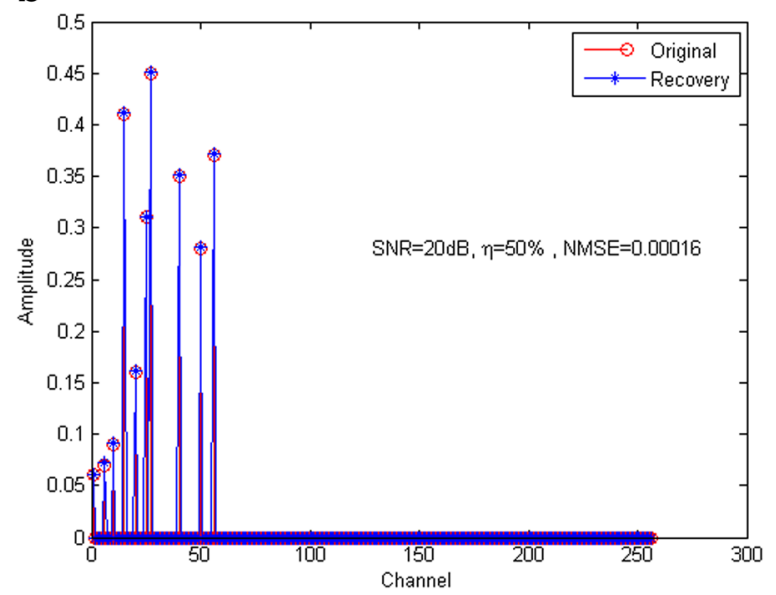

Fig. 5 Reconstruction signal of Toeplitz structure measurement matrix based on CC-S. a The result with $\eta=25 \%$. $\mathbf{b}$ The result with $\eta=50 \%$
Next, we simulated a scene with noisy signal reconstruction. We simulated the multipath channel and took the 12 paths with the largest channel gain in Fig. 5. In this experiment, $K=12$, the sampling rate is $\eta=25 \%, 50 \%$, and the SNR is set to $20 \mathrm{~dB}$. As can be seen in Fig. 5, the Toeplitz structure measurement matrix based on the CC-S can reconstruct the original signal, and the reconstruction error decreases with the increase of the sampling rate.

In Fig. 6, we compared the signal reconstruction performance of the Toeplitz structure measurement matrix based on CC-S under different compression sampling rates and different SNR. As can be seen in Fig. 6, when the sampling rate continues to increase, the performance of signal reconstruction is constantly improving. In the same sampling rate, when the SNR is larger, the effect of compressed-sensing channel estimation becomes better.

At the last, we compared the signal reconstruction performance of the Toeplitz structure measurement matrix based on the complementary sequences and Gaussian random matrix under the same conditions. As can be seen in Fig. 7, the two matrices achieve approximate signal reconstruction performance regardless of the sampling rate of $25 \%$ or $50 \%$. As a PN sequence, the correlation function of CC-S has similar performance with the Gaussian sequence. However, the CC-S can be constructed more easily in practice.

\section{Conclusion}

Aiming at the measurement matrix design problem, this paper proposes the Toeplitz structure measurement matrix based on CC-S to solve the complexity problem of compressed sensing algorithms in a massive MIMO system, and it opens a new direction in the field of measurement matrix construction. Compared with the classical Gaussian random matrix, the proposed measurement matrix can reduce a lot of hardware resources due to the element of CC-S is deterministic. At the same time, the Spark characteristics of the

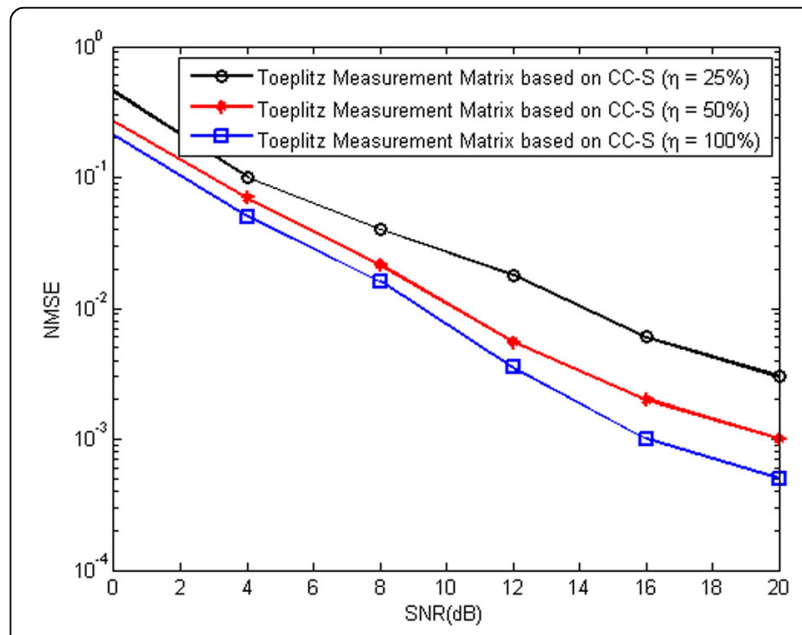

Fig. 6 Reconstruction performance analysis 


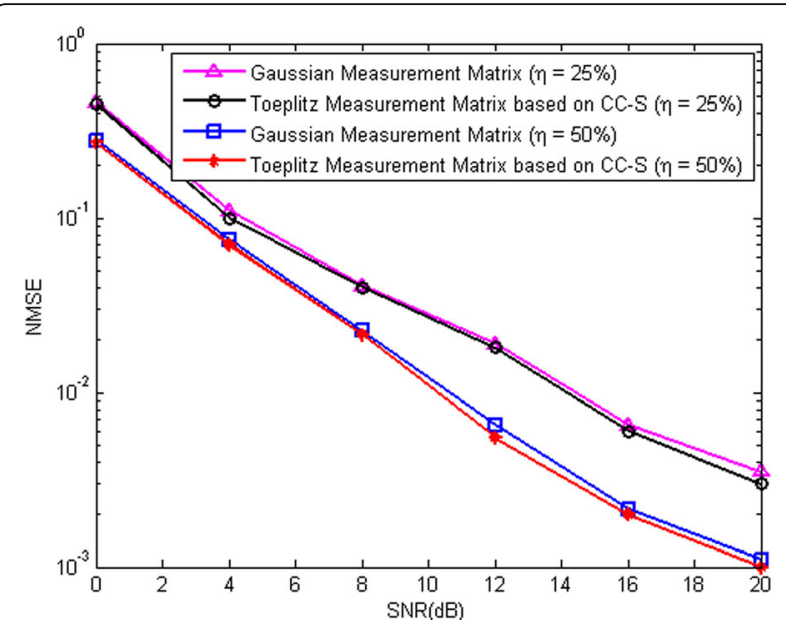

Fig. 7 Performance analysis between the Toeplitz measurement matrix and Gaussian random matrix

measurement matrix based on CC-S are proved. In addition, in terms of channel estimation performance, the Toeplitz structure measurement matrix based on CC-S can obtain nearly equivalent estimation performance with the Gaussian random matrix, but its generation and complexity are lower when applied to the calculation in practice. However, the drawback of the CC-S is that the length is limited. The CC-S solution only exists for some restricted length. There are still many important technologies to be researched in a massive MIMO system, such as codebook design, angle domain channel analysis, and pilot pollution and so on.

\section{Appendix}

We decompose the URA to a sort of sub-array according to the $x$ axis as a referenced direction. While the array response of the first sub-array is denoted as $a_{x, T}\left(\theta_{\ell, T}\right.$, $\left.\phi_{\ell, T}\right)$. Then, the $n$th sub-array is formulized as $a_{x, T}\left(\theta_{\ell, T}\right.$, $\left.\phi_{\ell, T}\right) F_{y}^{n-1}$. Take the second sub-array as an example:

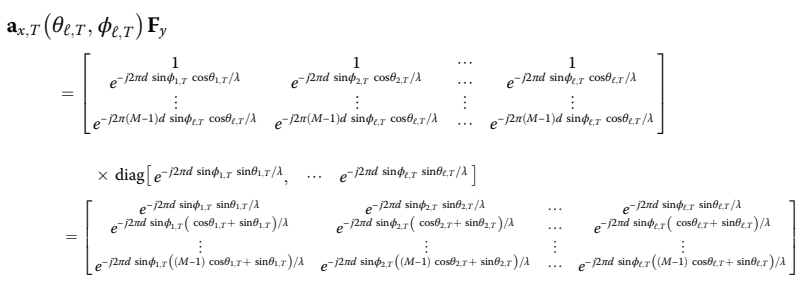

$\mathbf{a}_{x, T}\left(\theta_{\ell, T}, \phi_{\ell, T}\right) \mathbf{F}_{y}^{n-1} \in \mathbb{C}^{M \times L}$ is the second sub-array response along the horizontal direction. Besides, Eq. (17) can be also expressed as follows:
$\mathbf{A}_{T}=\left[\mathbf{a}_{x, T}\left(\theta_{\ell, T}, \phi_{\ell, T}\right), \quad \mathbf{a}_{x, T}\left(\theta_{\ell, T}, \phi_{\ell, T}\right) \mathbf{F}_{y}, \quad \cdots, \quad \mathbf{a}_{x, T}\left(\theta_{\ell, T}, \phi_{\ell, T}\right) \mathbf{F}_{y}^{N-1}\right]^{H} \in \mathbb{C}^{N_{\mathrm{BS}} \times L}$

As for Eq. (17):

$$
\begin{aligned}
& \mathbf{A}_{T}=\sum_{\ell=1}^{L} \mathbf{a}_{y, T}\left(\theta_{\ell, T}, \phi_{\ell, T}\right) \cdot \mathbf{a}_{x, T}\left(\theta_{\ell, T}, \phi_{\ell, T}\right) \\
& =\left[\mathbf{a}_{y, T}\left(\theta_{1, T}, \phi_{1, T}\right) \otimes \mathbf{a}_{x, T}\left(\theta_{1, T}, \phi_{1, T}\right), \cdots, \mathbf{a}_{y, T}\left(\theta_{\ell, T}, \phi_{\ell, T}\right) \otimes \mathbf{a}_{x, T}\left(\theta_{L, T}, \phi_{L, T}\right)\right] \in \mathbb{C}^{N_{\mathrm{BS}} \times L} \\
& =\left[\begin{array}{ccc}
1 \times \mathbf{a}_{x, T}\left(\theta_{1, T}, \phi_{1, T}\right) & \cdots & 1 \times \mathbf{a}_{x, T}\left(\theta_{L, T}, \phi_{L, T}\right) \\
e^{-j 2 \pi d \sin \phi_{1} \sin \theta_{1, T}} \times \mathbf{a}_{x, T}\left(\theta_{1, T}, \phi_{1, T}\right) & \cdots & e^{-j 2 \pi d \sin \phi_{\ell} \sin \theta_{\ell, T}} \times \mathbf{a}_{x, T}\left(\theta_{L, T}, \phi_{L, T}\right) \\
\vdots & \vdots & \vdots \\
e^{-j 2 \pi d(N-1) \sin \phi_{1} \sin \theta_{1, T \lambda}} \times \mathbf{a}_{x, T}\left(\theta_{1, T}, \phi_{1, T}\right) & \cdots & e^{-j 2 \pi d(N-1) \sin \phi_{\ell} \sin \theta_{\ell, T}} \times \mathbf{a}_{x, T}\left(\theta_{L, T}, \phi_{L, T}\right)
\end{array}\right] \\
& =\left[\begin{array}{ccc}
1 \times \mathbf{a}_{x, T}\left(\theta_{1, T}, \phi_{1, T}\right) & \cdots & 1 \times \mathbf{a}_{x, T}\left(\theta_{L, T}, \phi_{L, T}\right) \\
\mathbf{F}_{y}(1) \times \mathbf{a}_{x, T}\left(\theta_{1, T}, \phi_{1, T}\right) & \cdots & \mathbf{F}_{y}(L) \times \mathbf{a}_{x, T}\left(\theta_{\ell, T}, \phi_{\ell, T}\right) \\
\vdots & \vdots & \vdots \\
\mathbf{F}_{y}^{N-1}(1) \times \mathbf{a}_{x, T}\left(\theta_{1, T}, \phi_{1, T}\right) & \cdots & \mathbf{F}_{y}^{N-1}(L) \times \mathbf{a}_{x, T}\left(\theta_{L, T}, \phi_{L, T}\right)
\end{array}\right] \\
& =\left[\begin{array}{c}
\mathbf{a}_{x, T}\left(\theta_{\ell, T}, \phi_{\ell, T}\right) \\
\mathbf{a}_{x, T}\left(\theta_{\ell, T}, \phi_{\ell, T}\right) \mathbf{F}_{y} \\
\vdots \\
\mathbf{a}_{x, T}\left(\theta_{\ell, T}, \phi_{\ell, T}\right) \mathbf{F}_{y}^{N-1}
\end{array}\right] \in C^{N_{\mathrm{BS} \times L}}
\end{aligned}
$$

where the $F_{y}(\ell)$ denotes the $\ell$ th element of $F_{y}$, then we can get the data model as Eq. (17).

\section{Abbreviation}

AACF: Aperiodic auto-correlation function; ACCF: Aperiodic cross-correlation function; AOAs: Angles of arrival; AODs: Angles of departure; AWGN: Additive white Gaussian noise; BS: Base station; CC-S: Complete complementary sequence; CSI: Channel state information; i.i.d: Independent identically distributed; MIMO: Multiple-input multiple-output; mmWave: Millimeter-wave; MS: Mobile station; NMSE: Normalized mean square error; OMP: Orthogonal matching pursuit; RF: Radio frequency; RIP: Restricted isometry property; RMSE: Root mean square error; URAs: Uniform rectangular arrays

\section{Acknowledgements}

This work was supported by the National Nature Science Funding of China (NSFC): 61401407 and the Fundamental Research Funds for the Central Universities.

\section{Authors' contributions}

$\mathrm{SL}$ is the main author of the current paper. BS and $L J$ contributed to the conception and design of the study. MC and HW commented on the work. All authors read and approved the final manuscript.

\section{Funding}

Not applicable.

\section{Availability of data and materials \\ Not applicable.}

\section{Competing interests}

The authors declare that they have no competing interests.

\section{Author details}

${ }^{1}$ School of Information and Communication Engineering, Communication University of China, Beijing 100024, People's Republic of China. ${ }^{2}$ Department of Electrical Engineering and Computer Science, York University, Toronto, Canada.

Received: 28 October 2019 Accepted: 18 December 2019

Published online: 30 December 2019

\section{References}

1. B. Yang, Z. Yu, J. Lan, et al., Digital beamforming-based massive MIMO transceiver for $5 \mathrm{G}$ millimeter-wave communications. IEEE Transactions on Microwave Theory and Techniques 66(7), 3403-3418 (2018)

2. G. Kwon, N. Kim, H. Park, Millimeter wave SDMA with limited feedback: RFonly beamforming can outperform hybrid beamforming. IEEE Transactions on Vehicular Technology 68(2), 1534-1548 (2018) 
3. A. Ghosh, T.A. Thomas, M.C. Cudak, et al., Millimeter-wave enhanced local area systems: a high-data-rate approach for future wireless networks. IEEE Journal on Selected Areas in Communications 32(6), 1152-1163 (2014)

4. V. Raghavan, S. Subramanian, J. Cezanne, et al., Single-user versus multi-user precoding for millimeter wave MIMO systems. IEEE Journal on Selected Areas in Communications 35(6), 1387-1401 (2017)

5. A. Alkhateeb, J. Mo, N. Gonzalez-Prelcic, et al., MIMO precoding and combining solutions for millimeter-wave systems. IEEE Communications Magazine 52(12), 122-131 (2014)

6. R. Méndez-Rial, C. Rusu, N. González-Prelcic, et al., Hybrid MIMO Architectures for millimeter wave communications: phase shifters or switches? IEEE Access 4, 247-267 (2015)

7. W. Ma, C. Qi, Beamspace channel estimation for millimeter wave massive MIMO system with hybrid precoding and combining. IEEE Transactions on Signal Processing 66(18), 4839-4853 (2018)

8. J. Lee, G. Gil, Y.H. Lee, Exploiting spatial sparsity for estimating channels of hybrid MIMO systems in millimeter wave communications. 2014 IEEE Global Communications Conference (2014), pp. 3326-3331

9. H. Wang, W. Zhang, Y. Liu, et al., On design of non-orthogonal pilot signals for a multi-cell massive MIMO system. IEEE Wireless Communications Letters 4(2), 129-132 (2015)

10. A. Quayum, H. Minn, Y. Kakishima, Non-orthogonal pilot designs for joint channel estimation and collision detection in grant-free access systems. IEEE Access 6, 55186-55201 (2018)

11. P. Li, Channel estimation and signal reconstruction for massive MIMO with non-orthogonal pilots (IEEE Conference on Computer Communications Workshops (INFOCOM WKSHPS), Atlanta, GA, 2017), pp. 349-353

12. X. He, R. Song, W.P. Zhu, Pilot allocation for distributed compressed sensing based sparse channel estimation in MIMO-OFDM systems. IEEE Transactions on Vehicular Technology 65(5), 2990-3004 (2015)

13. A. Alkhateeb, O. El Ayach, G. Leus, et al., Channel estimation and hybrid precoding for millimeter wave cellular systems. IEEE Journal of Selected Topics in Signal Processing 8(5), 831-846 (2014)

14. H. Jarvis, U.B. Waheed, R. Gil, et al., Toeplitz compressed sensing matrices with applications to sparse channel estimation. IEEE Transactions on Information Theory 56(11), 5862-5875 (2010)

15. E. Candes, T. Tao, Decoding by linear programming. IEEE Transactions on Information Theory 51(12), 4203-4215 (2005)

16. E. Candes, J. Romberg, T. Tao, Stable signal recovery from incomplete and inaccurate measurements. Communications on Pure and Applied Math 59(8), 1207-1223 (2006)

17. Z. Gao, L. Dai, C. Hu, et al., Channel estimation for millimeter-wave massive MIMO with hybrid precoding over frequency-selective fading channels. IEEE Communications Letters 20(6), 1259-1262 (2016)

18. E. Candès, M. Wakin, An introduction to compressive sampling. IEEE Signal Processing Magazine 25(2), 21-30 (2008)

19. S.T. Xia, X.J. Liu, Y. Jiang, et al., Deterministic constructions of binary measurement matrices from finite geometry. IEEE Transactions on Signal Processing 63(4), 1017-1029 (2015)

\section{Publisher's Note}

Springer Nature remains neutral with regard to jurisdictional claims in published maps and institutional affiliations.

\section{Submit your manuscript to a SpringerOpen ${ }^{\circ}$ journal and benefit from:}

- Convenient online submission

- Rigorous peer review

- Open access: articles freely available online

- High visibility within the field

- Retaining the copyright to your article

Submit your next manuscript at $\boldsymbol{\nabla}$ springeropen.com 